\title{
Internet of things (IoT) and water crisis
}

\begin{abstract}
In the coming years, technology will impact the water crisis experience in many ways. Internet of Things (IoT) continues to confirm its important position in the context of Information and Communication Technologies and the development of society. The purpose of this proposing SMART2L is to find out the potential of IoT to be a part of the effort to curb the problem of water crisis and manage water resources efficiently. Further, efforts are necessary for releasing the full potential of IoT systems and technologies. Therefore, this paper presents a study about IoT that stands to change dramatically the way it impacts on the water crisis in Malaysia. On the other hand, IoT also brings tremendous challenges to water management in Malaysia. Hence, this paper also presents the perspective on the challenges of SMART2L in delivering its service to provide the assistance in solving water crisis especially in managing water resources.
\end{abstract}

Keyword: SMART2L; IoT; Communication; Water crisis; Water; Resources; Arduino 